Supporting Information

\title{
A PDMS-Paper Hybrid Lateral Flow Assay for Highly Sensitive Point-of-Care Nucleic Acid Testing
}

Jane Ru Choi ${ }^{1,2,3}$, Zhi Liu ${ }^{3,4}$, Jie Hu${ }^{1,3}$, Ruihua Tang ${ }^{1,3,5,6}$, Yan Gong ${ }^{1,3}$, Shangsheng Feng $^{3,7,8}$, Hui Ren ${ }^{3,9}$, Ting Wen ${ }^{10}$, Hui Yang ${ }^{5,6}$, Zhiguo Qu ${ }^{4}$, Belinda PingguanMurphy $^{2}$, Feng $\mathrm{Xu}^{1,3^{*}}$

${ }^{1}$ The Key Laboratory of Biomedical Information Engineering of Ministry of Education, School of Life Science and Technology, Xi' an Jiaotong University, Xi'an 710049, PR China.

${ }^{2}$ Department of Biomedical Engineering, Faculty of Engineering, University of Malaya, Lembah Pantai, 50603 Kuala Lumpur, Malaysia.

${ }^{3}$ Bioinspired Engineering and Biomechanics Center (BEBC), Xi'an Jiaotong University, Xi'an 710049, PR China.

${ }^{4}$ Key Laboratory of Thermo-Fluid Science and Engineering of Ministry of Education, School of Energy and Power Engineering, Xi'an Jiaotong University, Xi'an 710049, PR China.

${ }^{5}$ School of Life Sciences, Northwestern Polytechnical University, Xi'an 710072, PR China.

${ }^{6}$ Key Laboratory of Space Bioscience and Biotechnology, Northwestern Polytechnical University, Xi'an 710072, PR China.

${ }^{7}$ MOE Key Laboratory of Multifunctional Materials and Structures (LMMS), School of Aerospace, Xi'an Jiaotong University, Xi'an 710049, PR China.

${ }^{8}$ State Key Laboratory of Mechanical Structure Strength and Vibration, School of Aerospace, Xi'an Jiaotong University, Xi'an 710049, PR China.

${ }^{9}$ Department of Respiratory and Critical Care Medicine, The First Affiliated Hospital of Xi'an Jiaotong University, Xi'an 710061, PR China.

${ }^{10} X i$ 'an Diandi Biotech Company, Xi'an, PR China.

*Corresponding author: fengxu@mail.xjtu.edu.cn

\section{Table of contents}

$\begin{array}{ll}\text { Selection of an appropriate shunt material } & \mathrm{S}-2\end{array}$

$\begin{array}{ll}\text { Schematic of modified lateral flow strips } & \text { S-3 }\end{array}$

$\begin{array}{ll}\text { The average velocity at the test zone } & \text { S-4 }\end{array}$

Characterization of LFA incorporated with PDMS barrier $\quad$ S-5

$\begin{array}{ll}\text { Oligonucleotide sequences used in the study } & \text { S-6 }\end{array}$

HBV-positive clinical samples confirmed by qPCR $\quad$ S-7 

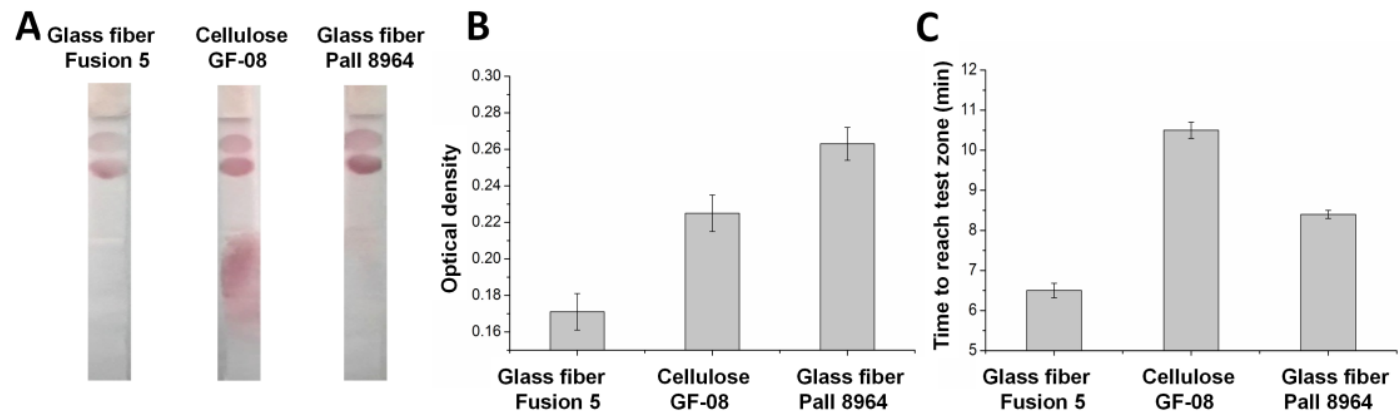

Supplementary Figure S1. Selection of an appropriate shunt material. (A-B)

Glass fiber Pall 8964 showed a significant higher optical density of the test zone $(0.225 \pm 0.01)$ with $(\mathbf{C})$ desirable duration required to reach the test zone as compared to that of Fusion 5 and Cellulose. 

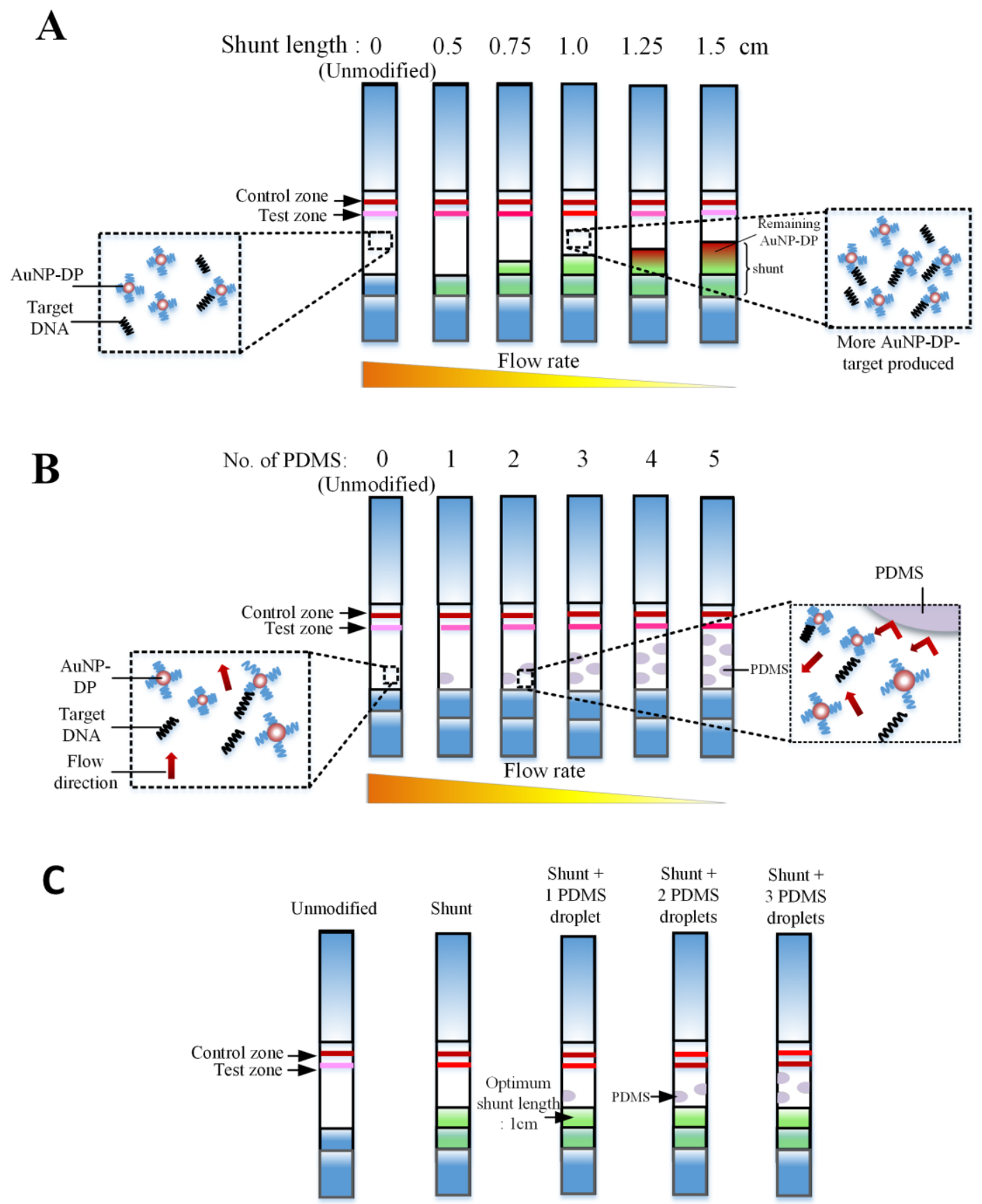

Flow rate

Supplementary Figure S2. Schematic of modified lateral flow strips. (A) Lateral flow strips with glass fiber shunt, (B) PDMS barrier and (C) the combination of both glass fiber shunt and PDMS barrier. 
A

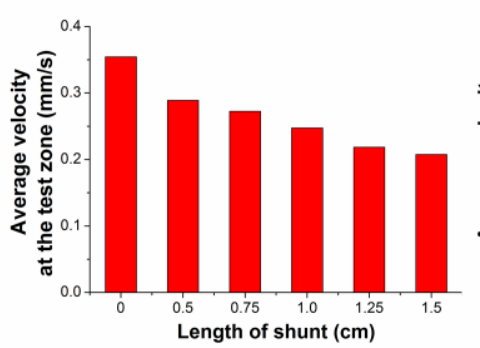

B

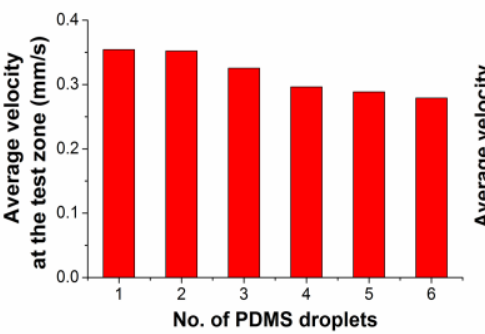

C

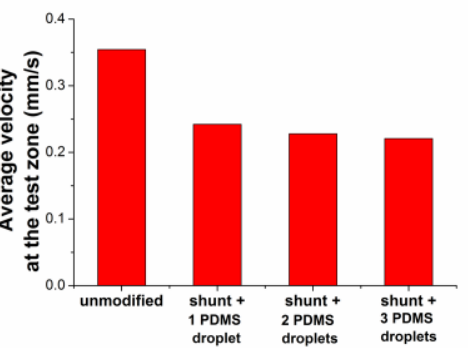

Supplementary Figure S3. The average velocity at the test zone. (A) The longer the length of the shunt, the lower the average velocity at the test zone. (B) The higher the number of PDMS droplets, the lower the average velocity at the test zone. $(\mathbf{C})$ The combination of both shunt and PDMS droplets decreases the average velocity at the test zone. 
A Unmodified nitrocellulose membrane

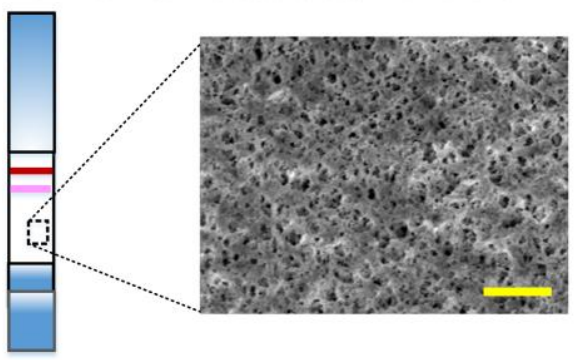

B

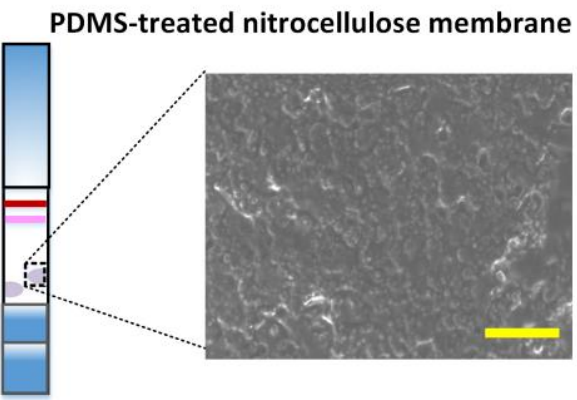

Supplementary Figure S4. Characterization of LFA incorporated with PDMS barrier. The SEM images of (i) unmodified nitrocellulose membrane and (ii) PDMS treated nitrocellulose membrane (Scale bars, $50 \mu \mathrm{m}$ ). 
Supplementary Table 1. Oligonucleotide sequences used in the study

\begin{tabular}{|c|c|}
\hline Name & Sequence (5’-3') \\
\hline HBV detector probe & $5^{\prime}$ - atgaatctggccacctgggt $-\left(\mathrm{CH}_{2}\right)_{6}-\mathrm{SH}-3^{\prime}$ \\
\hline HBV control probe & 5'- acccaggtggccagattcat /Biotin-3' \\
\hline HBV F3 & $5 '$ - cttctgtggagttactctctt-3' \\
\hline HBV B3 & $5^{\prime}$ - gctgactactaattcctgg-3' \\
\hline HBV FIP & 5'-Biotin/ ctcccgatacagagcagaggtttgccttctgacttctttcc-3' \\
\hline HBV BIP & 5 '- ttgttcacctcaccatacagcatgggtcttccaaattacttcc-3' \\
\hline HBV FLP & 5'- ggtgtcgaggagatctcgaata-3' \\
\hline HBV BLP & 5 '- attctgtgttggggtgagtt-3' \\
\hline HBV target DNA & 5 '- acccaggtggccagattcat -3 ' \\
\hline
\end{tabular}


Supplementary Table 2. HBV-positive clinical samples confirmed by qPCR

\begin{tabular}{ll}
\hline Sample & Concentration $(\mathbf{I U} / \mathbf{m L})$ \\
\hline $\mathrm{a}$ & $3.18 \times 10^{5}$ \\
$\mathrm{~b}$ & $7.33 \times 10^{6}$ \\
$\mathrm{c}$ & $1.96 \times 10^{5}$ \\
$\mathrm{~d}$ & $1.34 \times 10^{2}$ \\
$\mathrm{e}$ & $3.07 \times 10^{3}$ \\
$\mathrm{f}$ & $1.56 \times 10^{2}$ \\
$\mathrm{~g}$ & $2.72 \times 10^{4}$ \\
$\mathrm{~h}$ & $1.92 \times 10^{7}$ \\
$\mathrm{i}$ & $2.92 \times 10^{4}$ \\
$\mathrm{j}$ & $5.35 \times 10^{8}$ \\
$\mathrm{k}$ & $3.42 \times 10^{8}$ \\
$\mathrm{l}$ & $2.04 \times 10^{2}$ \\
$\mathrm{~m}$ & $2.64 \times 10^{3}$ \\
$\mathrm{n}$ & $7.07 \times 10^{6}$ \\
$\mathrm{o}$ & $2.72 \times 10^{4}$ \\
$\mathrm{p}$ & $1.59 \times 10^{2}$ \\
\hline
\end{tabular}

